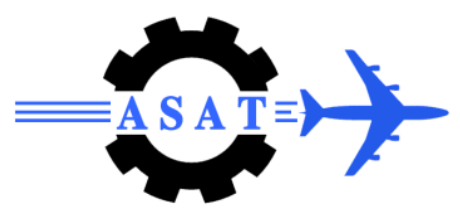

\title{
Optimization Using Genetic Algorithms of Aperiodic Linear Phased Arrays for Reduction of Grating Lobes for Wide Scanning
}

\author{
A.A. Omer ${ }^{*}$, H.H. Elbanna ${ }^{\dagger}$ and A.A. Mitkees
}

\begin{abstract}
The development of phased array antennas is challenged by the reduction of scan volume due to the appearance of grating lobes. The grating lobes appear in visible space as the spacing between elements be greater than half wavelength. The need for enlarging the spacing between elements is important either for reduction of mutual coupling or filling specific aperture size with small number of antenna elements. These grating lobes should be reduced or eliminated for enlarging the scan space. Nonuniform spacing between elements is an effective method for reduction of grating lobes as it breaks the periodicity of the array which is the reason of grating lobes. This method will be optimized using genetic algorithm implemented using MATLAB. The reduction is $-9.7 \mathrm{~dB}$ for 8 -elements and $-12.4 \mathrm{~dB}$ for 16 elements at $60^{\circ}$ scan angle.
\end{abstract}

Keywords: Aperiodic array, grating lobes, genetic algorithms, scan space.

\section{Introduction}

Most work is made on optimizing the array to have low side lobes at broadside [1]-[3]. As this array steered small angle from broadside, this side lobe level achieved at broadside will begin to increase as the steered angle increased. To have an array with low side lobe level at range of angles, the array should be optimized at the maximum desired scan angle [4]. Now if the array is steered between broadside and the maximum scan angle, the maximum side lobe level will be bounded by its value at the largest scan angle. This paper will convert the periodic array into aperiodic array in order to achieve this goal; the aperiodic array will be generated by disturbing the element positions of the periodic one. The optimization parameter will be the disturbance added to the spacing between elements in various ways to maintain low side lobe through scan to the largest scan angle. The optimization will be made at certain angle.

\section{Grating Lobes in the Periodic Array}

The maximum angle that the phased array allowed to scan without appearance of grating lobes is known from [5]

$$
\theta=\sin ^{-1}\left(\frac{\lambda}{d}-1\right)
$$

\footnotetext{
* Lecturer Assistant, BHIT, Benha University, atelep82@yahoo.com .

${ }^{\dagger}$ Egyptian Armed Forces, Egypt, hazem_elbanna@hotmail.com .

$¥$ Egyptian Armed Forces, Egypt, aamitkees@yahoo.com . 
where $\theta$ is the maximum scan angle from broadside, and $d$ is the uniform spacing between elements of the array. It is seen from this equation that if the array have uniform half wavelength spacing between elements, it will be able to scan the all range $\left(-90^{\circ}\right.$ to $\left.90^{\circ}\right)$ without appearance of grating lobes. But if the uniform spacing be grater than half wavelength the scan space will be significantly reduced. The array with 0.8 wavelengths uniform spacing between elements is allowed to scan up to $14.5^{\circ}$ from broadside without appearance of grating lobes according to equation (1). Even though the array with uniform spacing greater than half wavelength have grating lobes so it have small scan rang, it is very important because it is a simple solution for reduction of mutual coupling and have small number of elements for a given aperture so it will reducing the cost and overall weight of the antenna system.

\section{Aperiodic Phased Array}

To increase the scan space of the array of interelement spacing greater than half wavelength, the grating lobes must be eliminated or reduced. In order to achieve this, the periodic array should be replaced by aperiodic one as shown in figure 1 . This will be made by adding disturbances $\delta d_{i}$ to each interelement spacing.

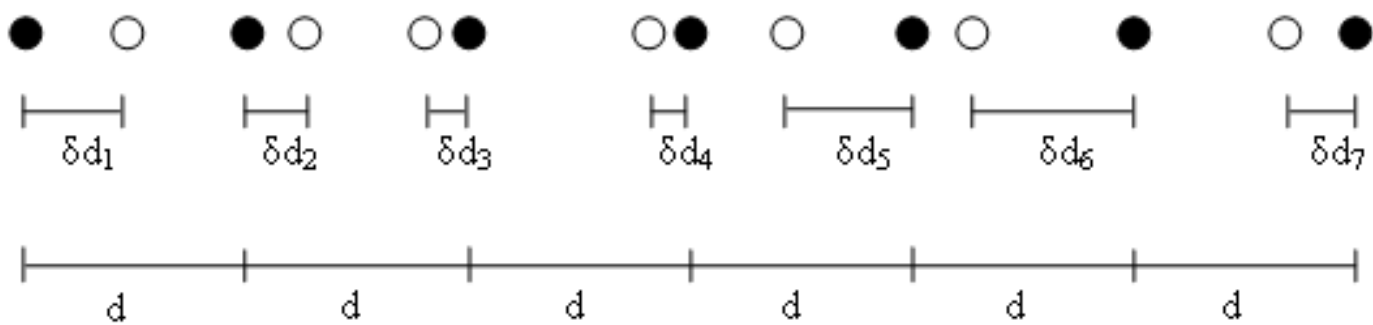

Fig. 1 Geometry of aperiodic array (hollow elements), which is created by adding disturbances to the position of each element in a periodic array (solid elements).

The array factor of the periodic array is given by

$$
S_{a}(\theta)=\sum_{i=1}^{\mathrm{K}} I_{n} e^{j \kappa_{o}(\mathrm{~K}-i) d\left[\sin (\theta)-\sin \left(\theta_{o}\right)\right]}
$$

where $\mathrm{K}$ is the total number of elements of the array, $d$ is the uniform spacing between elements, $I_{n}$ is the amplitude weight of the elements, and $\theta_{\mathrm{o}}$ is the steered angle.

The array factor of the aperiodic array made by adding the suitable element position disturbances $\delta d_{i}$ so that

$$
S_{a}(\theta)=\sum_{i=1}^{\mathrm{K}} I_{n} e^{j \kappa_{o} d_{i}\left[\sin (\theta)-\sin \left(\theta_{o}\right)\right]}
$$

where

$$
d_{i}=d(i-1)+\delta d_{i}
$$




\section{Genetic Algorithm}

A genetic algorithm is an iterative optimization algorithm prepared to emulate the processes of evolutionary biology. A block diagram for simple genetic algorithm is shown in figure 2 [3]. The genetic algorithm operators are crossover and mutation, our genetic algorithm use one point crossover and $20 \%$ mutation rate. This paper use population decimation as a selection strategy. The fitness of each individual will be evaluated using the fitness function which is defined by the maximum relative sidelobe level. The algorithm minimizes the fitness of the population.

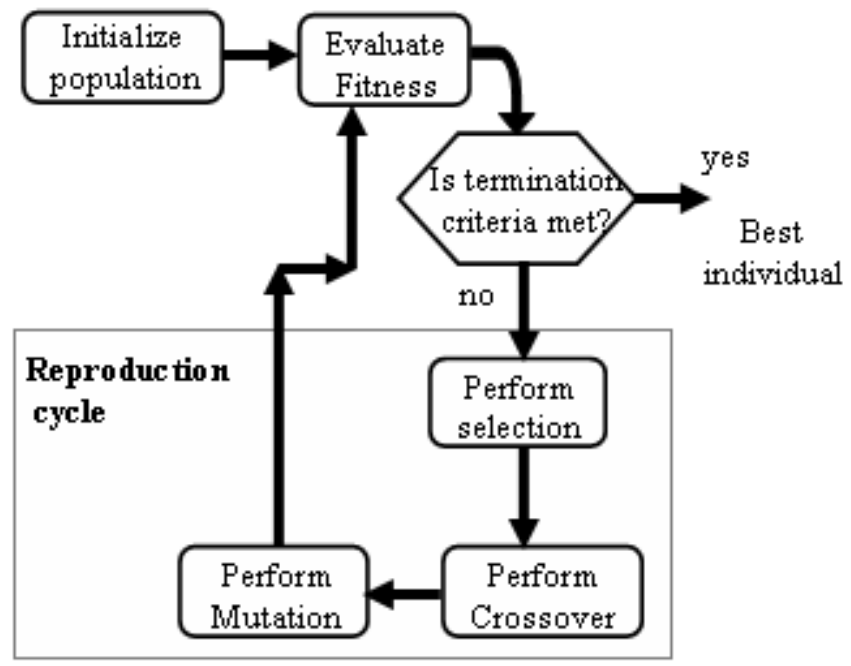

Fig. 2 A block diagram of a simple genetic algorithm optimizer

The genetic algorithm represent the phased array as a row of element position disturbances, this row will form the chromosome, this is what the genetic algorithm will optimized, certain number of chromosomes make the population [3]. The other parameters will be constant for a given optimization such as the number of elements of the array $\mathrm{K}$, the uniform spacing between elements $d$, and the maximum scan angle from broadside $\theta_{\mathrm{o}}$. The amplitude weight can leave constant or can be included for optimization for more little reduction.

The fitness function, equation (5), will be calculated from the array factor equation (3). Two restrictions have been made, the first restriction is on the total length of the array, equation (4), that is near the periodic one in order to keep the mainbeam width the same, the other is how much the element can be disturbed, it have a minimum value greater than zero in order to take the advantage of the disturbance, and maximum value smaller than $d$ uniform in order to keep a distance between elements, so a minimum distance between elements can be chosen according to mutual coupling allowed between elements.

$$
\begin{gathered}
\mathrm{ff}=\max \left(\frac{\sum_{i=1}^{\mathrm{K}} e^{j \kappa_{o} d_{i}\left[\sin (\theta)-\sin \left(\theta_{o}\right)\right]}}{\max (A F)}\right), \theta_{\text {ofist tight null }}<\theta<\theta_{\text {ofiss left null }} \\
, d_{i}=d(i-1)+\delta d_{i} \quad, 0<\delta d_{i}<d
\end{gathered}
$$

where $d$ is uniform spacing between elements, $\delta d_{i}$ disturbance of the element $i$ that will be optimized, $K$ is number of elements in the array, and $\theta_{0}$ angle that the array is steered from broadside. 


\section{Optimization Results}

An eight-element array with a uniform spacing of $0.8 \lambda$, a uniform current excitation and a scan angle of $60^{\circ}$ were chosen for the optimization. Maximum disturbance was chosen $0.4 \lambda$, so minimum spacing will be $0.4 \lambda$. The spacing out from genetic algorithm are shown in Table 1 , the geometry of this array is shown in figure 3 . Figure 4 shows the power radiation pattern of the array factor get out from the genetic algorithm in comparison to the periodic one, the optimization clearly reduces the grating lobes.

Table 1 The relative spacing between elements in terms of $\lambda$ for 8 -element array.

\begin{tabular}{l|c|c|c|c|c|c|c|c}
\hline \hline Element number & 1 & 2 & 3 & 4 & 5 & 6 & 7 & 8 \\
\hline Spacing & 0 & 0.404 & 0.473 & 1.232 & 0.517 & 0.947 & 0.439 & 0.953 \\
\hline \hline
\end{tabular}

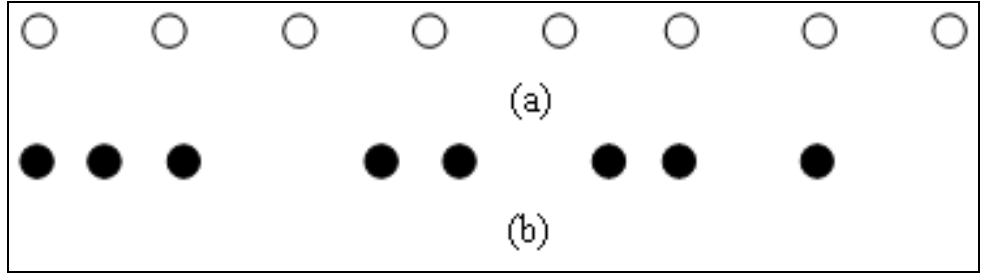

Fig. 3 a) The geometry of 8-element linear array with uniform spacing equal $0.8 \lambda$.

b) The geometry of the optimized array
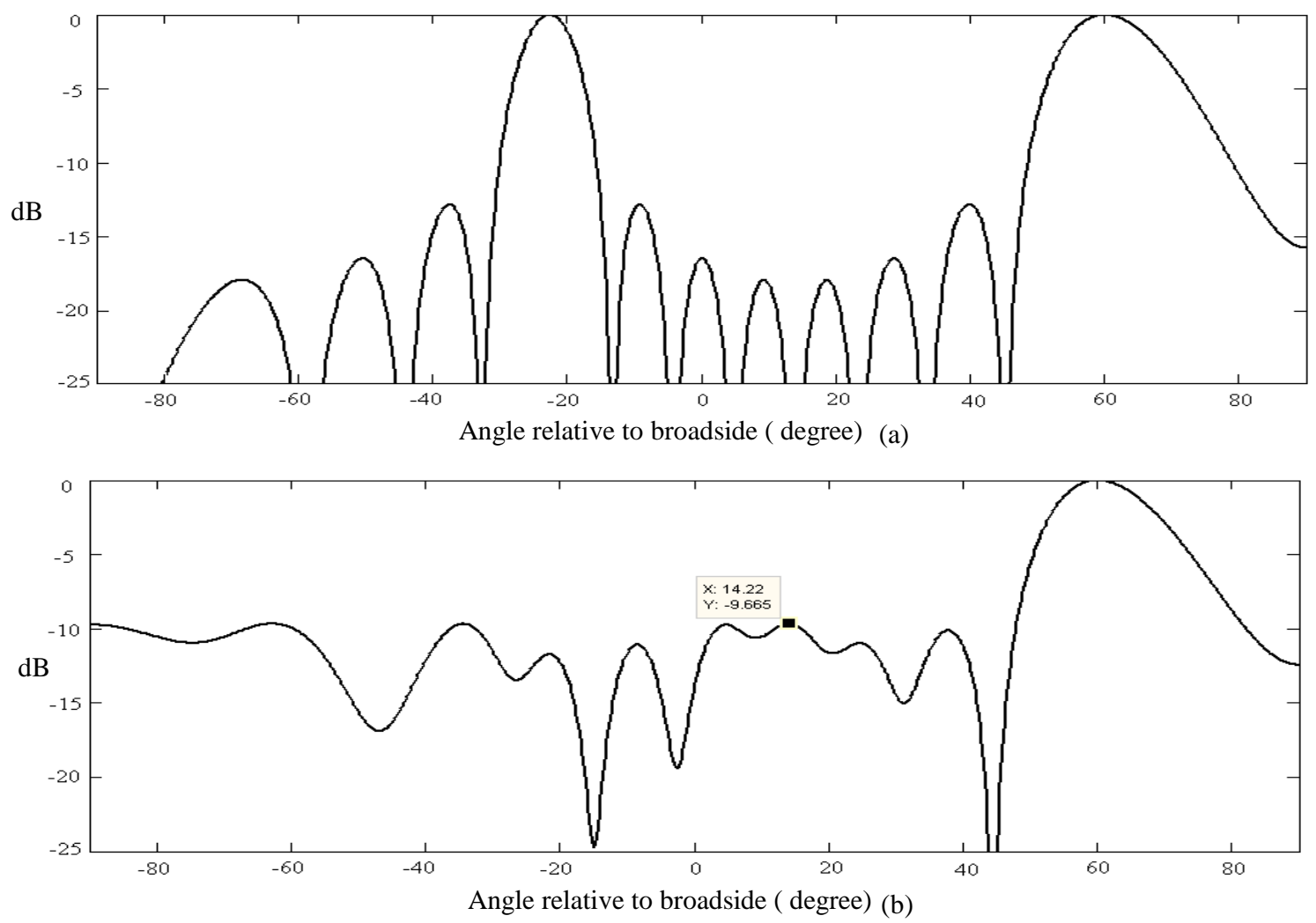

Fig. 4 (a) Power radiation pattern of the array factor of a linear 8-elements phased array antenna with a uniform spacing of $0.8 \lambda$ steered to $60^{\circ}$ (b) Power radiation pattern of the array factor of a linear 8-elements phased array antenna genetic algorithm optimized aperiodic array. 
More sidelobe reduction results when the number of elements is increased. By applying genetic algorithm on a 16-element array with a uniform spacing of $0.8 \lambda$, a uniform current excitation and maximum disturbance equal $0.45 \lambda$, so minimum spacing will be $0.35 \lambda$.

The sidelobes of the 16-element array are about 3-dB lower than the maximum sidelobe level for the eight-element array. The greater sidelobe suppression in the larger array is due to more degrees of freedom in choosing element positions [6]. The maximum sidelobe level in the optimized array is mainly dependent upon two factors, one of them is the number of the elements in the array and the second is the maximum steered angle. The maximum sidelobe level will remain constant at the value obtained from the genetic algorithm, as it is scanned from broadside to the optimization angle as shown in figure 5. The maximum sidelobe level increased as scanning above the optimization angle as shown in figure 6.
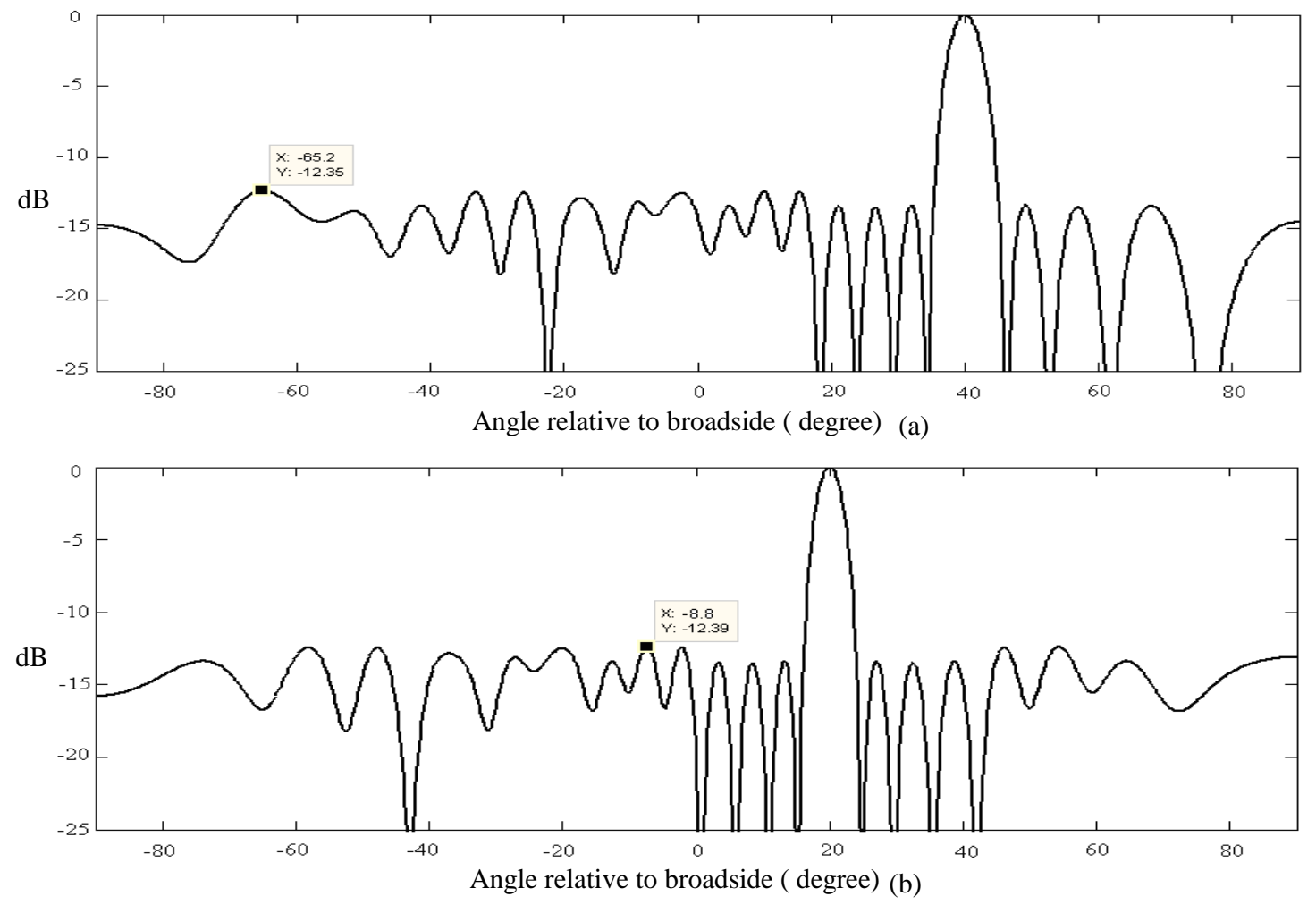

Fig. 5 Power radiation patterns of the array factor of a linear array steered from broadside to the optimization angle.

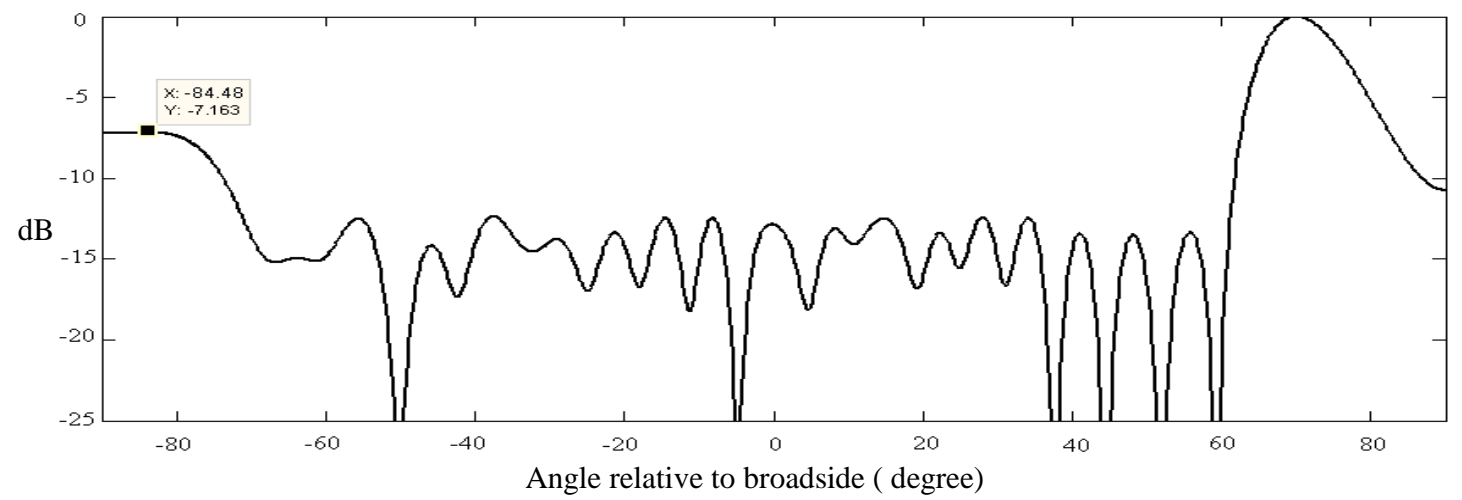

Fig. 6 Power radiation patterns of the array factor of a linear array steered above the optimization angle. 
The second factor that maximum side lobe level depends on is the maximum scan angle chosen; as the scan angle chosen was small the reduction will be better. For example, 24element array with uniform spacing of $0.8 \lambda$ scanned at $60^{\circ}$ from broadside, maximum disturbance equal $0.5 \lambda$, so minimum spacing will be $0.3 \lambda$. The reduction was $-13.47 \mathrm{~dB}$. Another example, 24-element array with uniform spacing of $0.8 \lambda$ scanned at $25^{\circ}$ from broadside the reduction was $-14.57 \mathrm{~dB}$, the reduction was better than steered angle of $60^{\circ}$ by $-1.1 \mathrm{~dB}$.

Figure 7 shows the maximum sidelobe level that can be achieved for three different sized arrays optimized from a $0.8 \lambda$ periodic array. These curves were generated by working many different genetic algorithms at scan angles from $0^{\circ}$ to $90^{\circ}$. Each point on a curve gives the maximum sidelobe level over the entire scan range of the array (up to and including the optimization angle). These curves show that the optimized aperiodic arrays were succeeded to have a larger scan volume than their periodic counterparts. The dotted line at $14.5^{\circ}$ represents the maximum possible scan angle of a periodic array without the appearance of grating lobes.

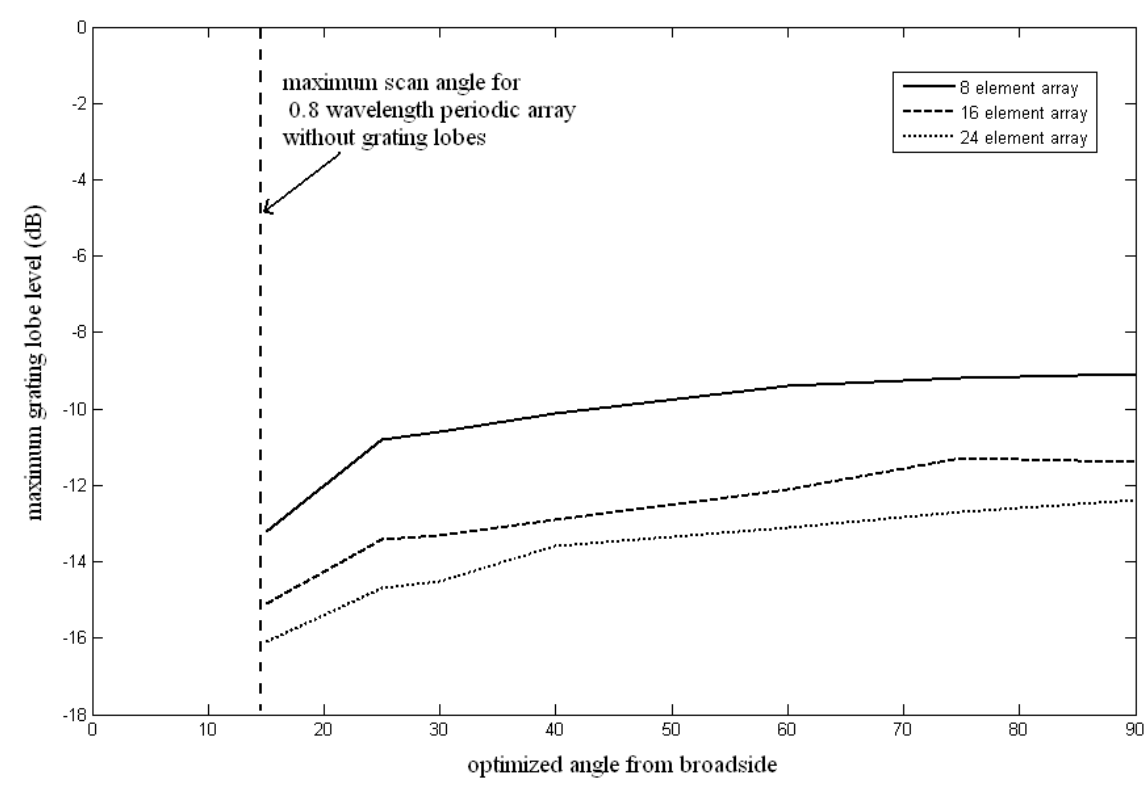

Fig. 7 Maximum grating lobe level that can be achieved over a range of different optimization angles.

Additional sidelobe suppression can be achieved by the inclusion of the amplitude of element excitation into the genetic algorithm; the improvement will be small because the grating lobes come mainly from the periodicity of the array.

Our results different from results obtained by reference [6] for angles above $60^{\circ}$ for 8 -element array and angles above $65^{\circ}$ for 16 and 24-elemet array, there results are shown in figure 8 . Reference [7] reports this error made by reference [6]. This error may be due to using a simple genetic algorithm.

\section{Conclusions}

For linear array with spacing between elements larger than half wavelength, a method of developing aperiodic phased arrays will reduce grating lobes when steered over large-scan angles. The aperiodic arrays were optimized via a genetic algorithm for a given scan angle and were shown to be steerable with constant sidelobe levels up to and including that angle. It was also shown that by increasing the size of the array the reduction can be increased at the same scan angle, the reduction is $-9.7 \mathrm{~dB}$ for 8 -elements and $-12.4 \mathrm{~dB}$ for 16 -elements at $60^{\circ}$ 
scan angle. Decreasing the scan angle also affect the reduction, the reduction is $-11.3 \mathrm{~dB}$ for 8-elements and $-13.4 \mathrm{~dB}$ for 16 -elements at $25^{\circ}$ scan angle.

It is also shown that for more reduction amplitude weights at elements will be optimized with the spacing, the improvement was $-1 \mathrm{~dB}$, this improvement is small because the grating lobes come mainly from the periodicity of the array.

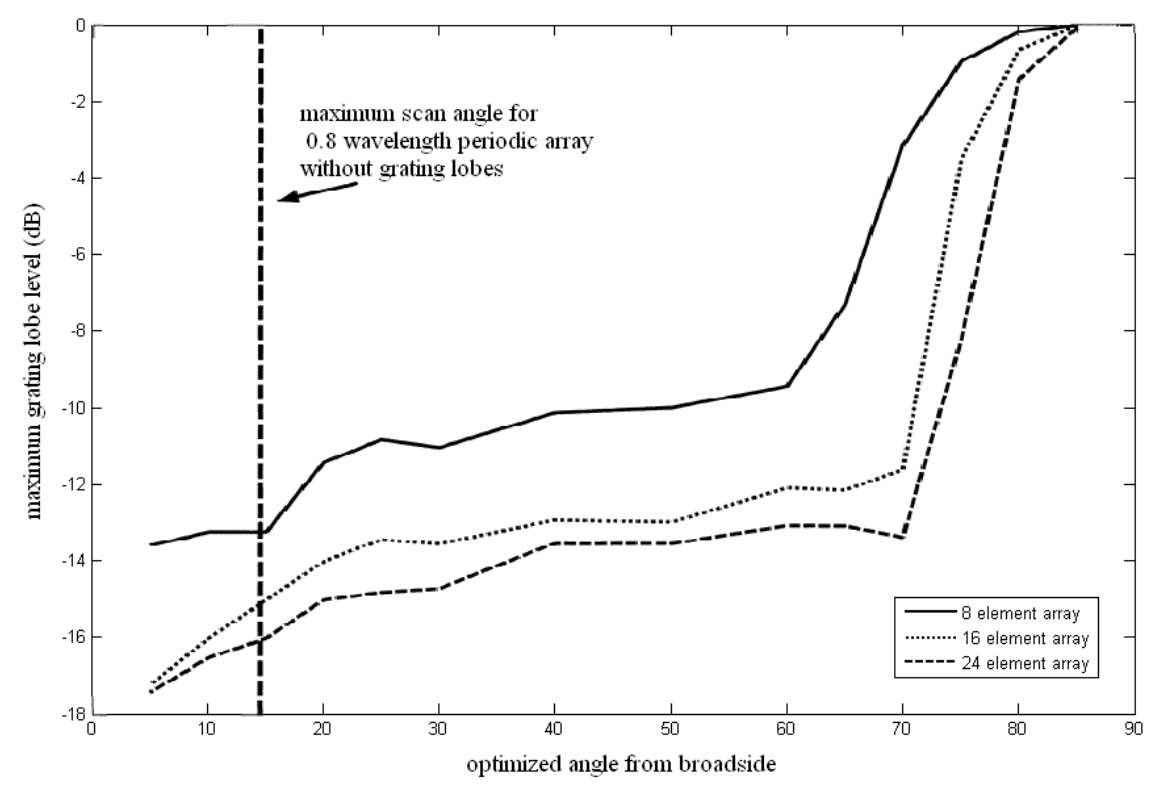

Fig. 8 Maximum grating lobe level that was achieved by ref. [6].

\section{References}

[1] R. L. Haupt, "Thinned Arrays Using Genetic Algorithms," IEEE Trans.Antennas Propagat., vol. 42, pp. 993-999, July 1994.

[2] R. L. Haupt, "An Introduction to Genetic Algorithms for Electromagnetics," IEEE Antennas Propagat. Mag., vol. 37, pp. 7-15, Apr. 1995.

[3] J. M. Johnson, Y. R. Samii, "Genetic Algorithms in Engineering Electromagnetics," IEEE Antennas Propagat. Mag., vol. 39, pp. 7-21, Aug. 1997.

[4] B. K. Chang, X. Ma, and H. B. Sequeira, "Minimax-Maxmini: A New Approach to Optimization of The Thinned Antenna Arrays," in Proc. IEEE Antennas and Propagation Society Int. Symp., vol. 1, Seattle,WA, 1994, pp. 514-517.

[5] Hubregt J. Visser, Array and Phased Array Antenna Basics, John Wiley \& Sons, New York, 2005.

[6] M. G. Bray, D. H. Werner, D. W. Boeringer, and D. W. Machuga, "Optimization of Thinned Aperiodic Linear Phased Arrays Using Genetic Algorithms to Reduce Grating Lobes During Scanning", IEEE Trans. Antennas Propag., vol. 50, pp. 1732-1742, Dec. 2002.

[7] W. C. Barott and P. G. Steffes "Grating Lobe Reduction in Aperiodic Linear Arrays of Physically Large Antennas," IEEE Antennas and Wireless Propagation Letters, vol. 8, pp. 406 - 408, May 2009. 